\title{
Acute Bacterial Meningitis Complicated by Brain Herniation Shortly After Lumbar Puncture: A Case Report
}

\author{
Khalid Ibrahim Al Noaim ${ }^{1}$ \\ ${ }^{1}$ Pediatric Department, College of Medicine, King Faisal University, Hofuf, Saudi Arabia \\ Correspondence: Pediatric Department, College of Medicine, King Faisal University, Hofuf, Saudi Arabia. E-mail: \\ n3aaim@hotmail.com
}

Received: January 8, 2018 Accepted: January 31, 2018 Online Published: February 12, 2018

doi:10.5539/gjhs.v10n3p121 URL: https://doi.org/10.5539/gjhs.v10n3p121

\begin{abstract}
Introduction: Brain herniation is a known complication of acute bacterial meningitis. Brain computed tomography $(\mathrm{CT})$ is requested before the lumbar puncture (LP) to rule out increased intracranial pressure. Delay in antibiotic administration, secondary to brain CT, leads to a poor clinical outcome.

Case Profile: A 10-year-old boy, who had a history of skull fracture and pneumococcal meningitis, was presented to emergency room (ER) with a history of fever, headache and vomiting for 2 days. Clinically, he was fully conscious and his vital signs were stable, with positive meningeal signs. Other systemic examinations were normal. Brain CT was normal before LP. LP suggested meningitis in the form of leukocytosis with low glucose and high protein. Antibiotics were adminestered after LP. Two hours after LP, the patient had deteriorations in the level of consciousness; respiratory arrest was followed by a coma. Brain CT was repeated which showed cerebellar herniation and subarachnoid hemorrhage. Neuroprotective strategy was performed without improvements. Blood and CSF cultures showed pneumococcal growth. After one week, brain death was confirmed.

Conclusion: Brain herniation is a severe complication of bacterial meningitis. Clinical findings are the best indicators to delay LP and predict the risk of herniation. Normal brain CT does not necessarily mean LP is safe. Early antibiotic administration improves mortality and morbidity. Unnecessary CT before LP leads to a delay in antibiotic administration and poor outcome.
\end{abstract}

Keywords: meningitis, herniation, lumber puncture, brain, fulminant

\section{Background}

Acute bacterial meningitis is a serious infection with a significant risk of a CNS sequel or mortality (Joffe, 2007; Swanson, 2015). Brain herniation is a known complication of acute bacterial meningitis (Joffe, 2007; Swanson, 2015). The most severe type of bacterial meningitis is fulminant meningitis (FM) (Radetsky, 2014). Computed tomography (CT) is requested to rule out increased intracranial pressure before the lumbar puncture (LP) (Swanson, 2015). There is no clear guideline about the indications of CT before LP in pediatric; there are different protocols for adults varying in accordance with the conservation (April, Long, \& Koyfman, 2017; Glimåker, Sjölin, Åkesson, \& Naucler, 2017). There is clear evidence that CT scan before LP leads to a delay in antibiotic administration (Nagra, Wee, Short, \& Banerjee, 2011; Glimaker, Johansson, Grindborg, Bottai, Lindquist, \& Sjolin, 2015). The delaying of antibiotic administration by more than 3 hours leads to a poor clinical outcome (Glimaker et al., 2015; Auburtin et al., 2006).

We presented a case of acute bacterial meningitis that had a normal brain CT prior to LP, which complicated by brain herniation within 2 hours after LP.

\section{Case Profile}

A 10-year-old boy was not known to have chronic illness. He was presented to the emergency department (ER) at 00:15 with a complaint of fever, headache and neck stiffness and, recurrent vomiting for 2 days, which is associated with photophobia and phonophobia. He was seen in a private clinic a day before and administered oral antibiotics. No history of change in the level of consciousness and no history of seizure or abnormal posture was recoded. He had a history of a skull fracture more than a year earlier and a history of pneumococcal meningitis 4 months earlier, which was treated with a full course of IV antibiotics. He was fully vaccinated. No past history of other recurrent infections and no family history of immunodeficiency was observed. In ER, he presented walking, 
fully conscious and oriented. Vital signs showed a temperature of $36.1^{\circ} \mathrm{C}$, heart rate of $111 \mathrm{~b} / \mathrm{min}$, respiratory rate of 28breaths/min, and BP: $115 / 77 \mathrm{mmHg}$. Neurological examination showed GCS 15/15 with positive meningeal signs. He had normal cranial nerves, tone, power and reflexes. Fundoscopy was not conducted. ENT examination showed congested throat. Other systemic examinations were normal. The CT scan performed before the LP at 02:30 was normal (Figure 1). LP was carried out at 03:30, which showed leukocytosis (WBC level 906/ $\mathrm{mm}^{3}$ with $58 \%$ neutrophils), protein $1.17 \mathrm{~g} / 1$ and glucose $1.3 \mathrm{mmol} / \mathrm{l}$. Blood CBC showed WBC 35000 (77\% neutrophils), hemoglobin $11.8 \mathrm{~g} / \mathrm{dl}$, and platelets 397000 . Serum chemistries (including $\mathrm{Na}, \mathrm{K}, \mathrm{Cl}, \mathrm{BUN}$, creatinine and CO2) were normal. At 04:15, he received ceftrixon and vancomycin. At 05:20, he developed irregular respiration and showed a decrease in the level of consciousness without any response to stimulation. CNS examination showed GCS: $3 / 15$ and bilateral fixed dilated pupils. He was intubated immediately and started on a neuroprotective strategy with hypertonic saline and mannitol infusions. No improvements were recorded. The CT scan was repeated after stabilization at 14:30 which showed cerebellar herniation and subarachnoid hemorrhage (Figure 2). Blood and CSF cultures were positive for Streptococcus pneumoniae. After one week, brain death was confirmed.

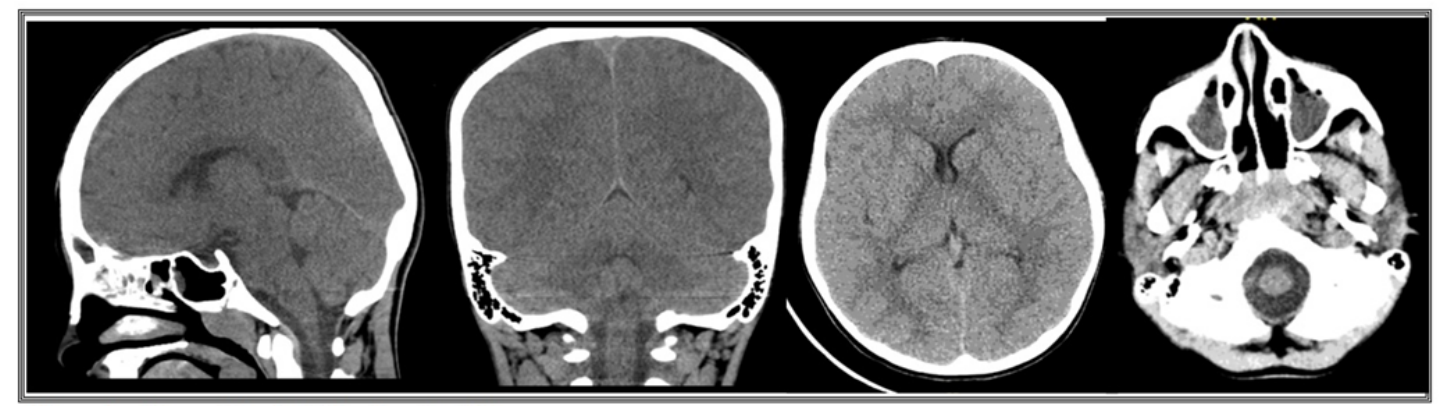

Figure 1. Normal brain CT before the LP

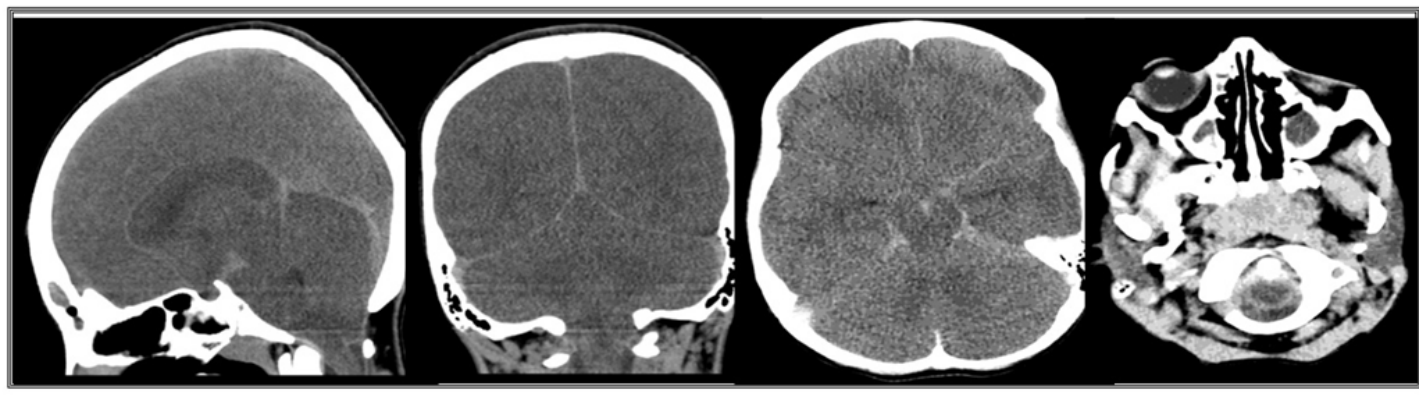

Figure 2. Cerebellar herniation with subarachnoid hemorrhage after the LP

\section{Discussion}

The child in the case suddenly deteriorated within 2 hours, from being fully conscious to falling into a coma. The causes of the deterioration will be discussed separately in detail. They could be either LP related, as the condition deteriorated shortly after LP, or a part of the meningitis process. The previous skull fracture is the cause of the recurrent pneumococcal meningitis (Ghosh, 2017).

Brain herniation affects around $5 \%$ of the cases of acute bacterial meningitis and accounted for $32 \%$ of its mortality (Joffe, 2007). Joffe found that there is a relation between the LP and brain herniation in his review, which showed brain herniation occurred within 3 hours from LP in 38\%, 4 to 12 hours in $41 \%$ and more than 12 hours in $21 \%$. Brain herniation occurred before LP in $11 \%$ of the cases which is against the risk of LP (Joffe, 2007). LP does not necessarily have to be the main cause, but may accelerate the process in the patient who ran the risk of herniation (Joffe, 2007; Swanson, 2015; Radetsky, 2014). Brain CT scan cannot totally rule out increased intracranial pressure (ICP). Rennick reported 5 out of 14 cases (36\%) of brain herniation in children with meningitis had normal CT prior to LP (Rennick, Shann, \& de Campo, 1993). Kastenbauer reported that 8 out of 10 cases (80\%) of brain herniation in adults with meningitis had normal CT prior to LP (Kastenbauer, Winkler, \& Pfister, 2002). Baker reported 6 out of 13 cases (46\%) of high ICP measured by LP had normal CT (Baker et al., 1994). A normal 
CT scan does not mean LP is totally safe. There is clear evidence that the brain CT scan before LP leads to a delay in antibiotic administration (Nagra, 2011; Glimaker et al., 2015). The delaying of antibiotic administration for more than 3 hours leads to a poor clinical outcome (Glimaker et al., 2015; Auburtin et al., 2006). Most of the case reports and case series of brain herniation secondary to meningitis showed change in the level of consciousness before LP (Joffe, 2007). The clinical contraindications of LP are changing levels of consciousness, fixed dilated pupils, fixed eye deviation, posturing, respiratory changes, papilledema, recent seizure or hypertension with bradycardia (Joffe, 2007; Swanson, 2015; Glimaker, 2015; Auburtin et al., 2006). We conclude that the clinical signs and symptoms are the most important indicators in identifying the risk of herniation and deciding when to delay LP. A cohort prospective study showed a more restricted guideline to perform brain CT before LP had less mortality and more favorable outcome due to earlier antibiotics administrations (Glimåker et al., 2017). The practice of brain CT before LP should be restricted to specific situations, where ICP is suspected clinically. The CSF culture will not be affected if the antibiotics are administered within 4 hours and before LP (Michael et al., 2010).

Bacterial meningitis is either acute, subacute or fulminant. Fulminant meningitis (FM) is the most severe type that causes abrupt developments of herniation or death within 48 hours from the onset of symptoms (Radetsky, 2014). A reported case of FM showed a sudden deterioration in ER within 2 hours to a coma and brain herniation without undergoing LP. The most common pathogen is pneumococcal infection. Most of the reported cases had normal brain CT before LP and diagnosed as FM retrospectively (Radetsky, 2014). Herniation in FM is unresponsive to the interventions, but showed a response in acute condition (Joffe, 2007; Radetsky, 2014). Mortality occurred among more than 50\% (Radetsky, 2014).

In our case, it is still a challenge to determine the exact cause of the deterioration. No one can totally detemine the cause of this deterioration. The patient did not have any clinical contraindications of LP with the normal brain CT, which made LP less likely to be the cause. The sudden deterioration was in favor of acute or fulminant meningitis processes, especially since the case was associated with a delaying of antibiotic administration secondary to performing CT and LP.

\section{Conclusion}

Brain herniation is a severe complication of bacterial meningitis. Clinical findings are the best indicators of delaying LP and predicting the risk of herniation. Normal brain CT does not necessarily mean LP is safe. Early antibiotic administration improves mortality and morbidity. Unnecessary CT before LP leads to a delay in antibiotics administration and poor outcome.

\section{Competing Interests Statement}

The authors declare that they has no competing or potential conflicts of interest.

\section{References}

April, M., Long, B., \& Koyfman, A. (2017). Emergency Medicine Myths: Computed Tomography of the Head Prior to Lumbar Puncture in Adults with Suspected Bacterial Meningitis - Due Diligence or Antiquated Practice?. The Journal of Emergency Medicine, 53(3), 313-321. https://doi.org/10.1016/j.jemermed.2017.04.032

Auburtin, M., Wolff, M., Charpentier, J., Varon, E., Le Tulzo, Y., Girault, C., ... Timsit, J. (2006). Detrimental role of delayed antibiotic administration and penicillin-nonsusceptible strains in adult intensive care unit patients with pneumococcal meningitis: The PNEUMOREA prospective multicenter study. Critical Care Medicine, 34(11), 2758-2765. https://doi.org/10.1097/01.CCM.0000239434.26669.65

Baker, N. D., Kharazi, H., Laurent, L., Walker, A. T., Williamson, D. S., Weissman, B. N., ... Sanchez, R. (1994). The efficacy of routine head computed tomography (CT scan) prior to lumbar puncture in the emergency department. J Emerg Med., 12, 597-601. https://doi.org/10.1016/0736-4679(94)90409-X

Ghosh, A. (2017). Diagnostic Dilemma in a 15-Year-Old Boy with Recurrent Pneumococcal Meningitis. Journal of Clinical And Diagnostic Research. https://doi.org/10.7860/JCDR/2017/26456.9875

Glimaker, M., Johansson, B., Grindborg, O., Bottai, M., Lindquist, L., \& Sjolin, J. (2015). Adult Bacterial Meningitis: Earlier Treatment and Improved Outcome Following Guideline Revision Promoting Prompt Lumbar Puncture. Clinical Infectious Diseases, 60(8), 1162-1169. https://doi.org/10.1093/cid/civ011

Glimåker, M., Sjölin, J., Åkesson, S., \& Naucler, P. (2017). Lumbar Puncture Performed Promptly or After Neuroimaging in Acute Bacterial Meningitis in Adults: A Prospective National Cohort Study Evaluating Different Guidelines. Clinical Infectious Diseases. https://doi.org/10.1093/cid/cix806 
Joffe, A. (2007). Lumbar Puncture and Brain Herniation in Acute Bacterial Meningitis: A Review. Journal of Intensive Care Medicine, 22(4), 194-207, https://doi.org/10.1177/0885066607299516

Kastenbauer, S., Winkler, F., \& Pfister, H. W. (2002). Cranial CT before lumbar puncture in suspected meningitis. N Engl J Med., 346, 1248-1249. https://doi.org/10.1056/NEJM200204183461615

Michael, B., Menezes, B., Cunniffe, J., Miller, A., Kneen, R., Francis, G., ... Solomon, T. (2010). Effect of delayed lumbar punctures on the diagnosis of acute bacterial meningitis in adults. Emergency Medicine Journal, 27(6), 433-438. https://doi.org/10.1136/emj.2009.075598

Nagra, I., Wee, B., Short, J., \& Banerjee, A. (2011). The role of cranial CT in the investigation of meningitis. JRSM Short Reports, 2(3), 1-9. https://doi.org/10.1258/shorts.2011.010113

Radetsky, M. (2014). Fulminant Bacterial Meningitis. The Pediatric Infectious Disease Journal, 33(2), 204-207. https://doi.org/10.1097/01.inf.0000435508.67490.f0

Rennick, G., Shann, F., \& de Campo, J. (1993). Cerebral herniation during bacterial meningitis in children. BMJ, 306(6883), 953-955. Retrieve from https://www.ncbi.nlm.nih.gov/pmc/articles/PMC1677428/

Swanson, D. (2015). Meningitis. Pediatrics in Review, 36(12), 514-526. https://doi.org/10.1542/pir.36-12-514

\section{Copyrights}

Copyright for this article is retained by the author(s), with first publication rights granted to the journal.

This is an open-access article distributed under the terms and conditions of the Creative Commons Attribution license (http://creativecommons.org/licenses/by/4.0/). 